Canadian Oncology

Nursing Journal

Revue canadienne

de soins infirmiers

en oncologie

Volume 31, Issue 1 • Winter 2021

elSSN: 2368-8076 


\section{Building competency in hematopoietic stem cell transplant coordination: Evaluating the effectiveness of a learning pathway for nurses new to this role}

by Cheryl Page, Charissa Cordon, and Jiahui Wong

\begin{abstract}
There has been an increased need for highly skilled nursing staff trained in hematopoietic stem cell transplant (HSCT) care driven by the increase in transplant eligible patients (CCO, 2017). There is a lack of literature pertaining to orientation to HSCT coordination, a highly specialized nursing role. Historically, orientation to HSCT coordination has been preceptor based without a formal orientation process.
\end{abstract}

Objectives: For this pilot study, a learning pathway and educational tools were developed and evaluated to support a standardized and systematic approach to the staffeducation and to improve quality of care.

\section{AUTHOR NOTES}
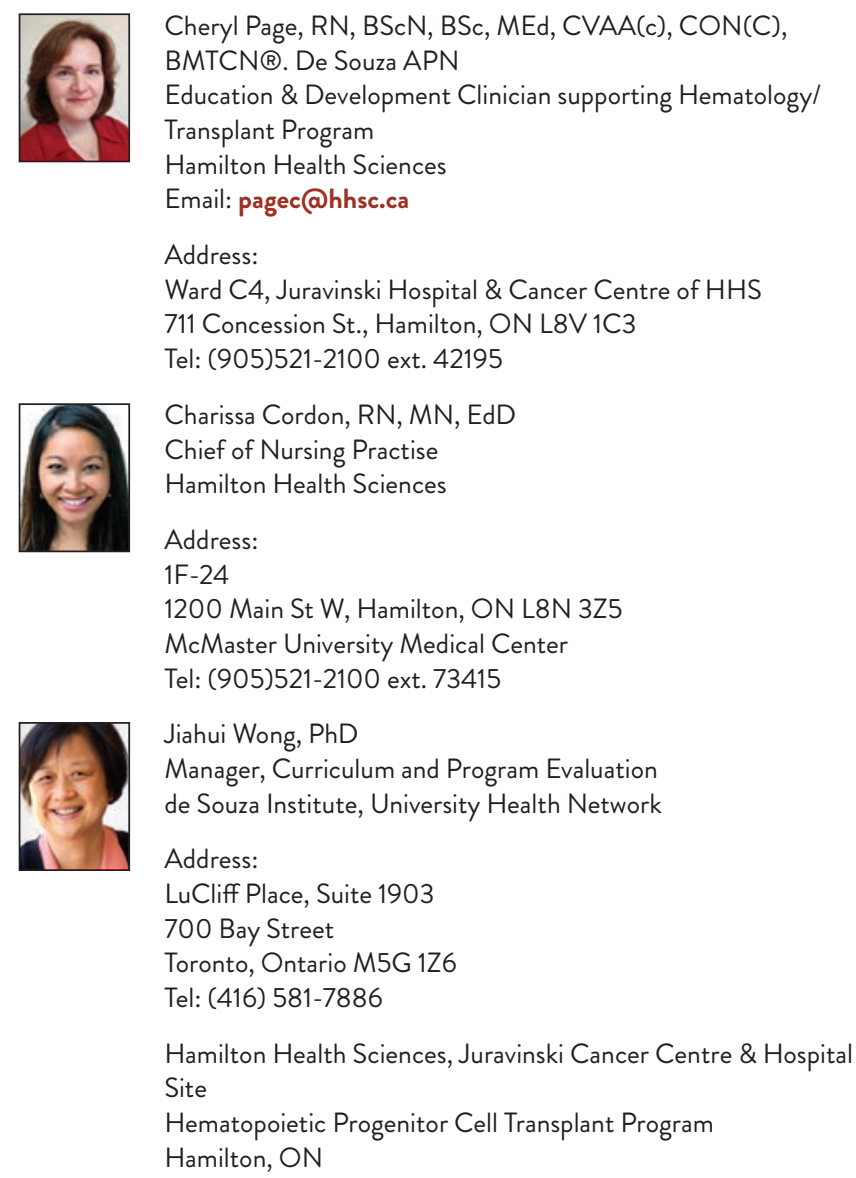

Methods: Eight nurses new to HSCT coordination participated in this study and completed the follow-up evaluations. Nurses were assessed before the intervention to identify knowledge gaps for each sub-role of allogeneic and autologous stem cell coordination. Following the assessment, nurses received a tailored self-directed learning package mapped to a learning pathway and a competency evaluation tool. A competency-based "building-block" training approach was used in which a new skill was added only after a previous skill was mastered. Utilizing the Kirkpatrick (2006) model, nurses were evaluated pre- and postorientation to assess changes in both knowledge and in behaviour relating to transplant coordination.

Results: Participants reacted favourably to these tools and reported a significantly higher level of knowledge and competency in the transplant coordination role following orientation. Further research on the use of a learning pathway to guide the orientation of nurses to the HSCT coordination role would complement this pilot study.

\section{INTRODUCTION}

There has been an increase in the need for hematopoi1 etic stem cell transplant (HSCT) trained nurses due to a critical increase in the number of patients eligible for transplants. This increase in eligible patients was the result of multiple factors including advances in technology, expanding indications for transplant, expanded eligibility criteria, and Ontario's aging population. During 2016 and 2017, 913 patients received HSCT within Ontario and an additional 45 patients received out-of-country transplants (CCO, 2017). This represents a 79 percent increase from 2009-10 (CCO, 2017). This rate of increase is expected to accelerate. In preparation for this rise, many hospitals that offer hematopoietic progenitor cell transplants, including Hamilton Health Sciences (HHS), are improving models of care and increasing training for staff who are new to this area. HHS is one of three hospitals in Ontario that currently offers patients all three types of transplants (autologous, allogeneic related donor, and allogeneic unrelated donor). Each of these centres is accredited by the Foundation for Accreditation for Cellular Therapy (FACT).

Allogeneic peripheral blood stem cell transplantation involves the use of chemotherapy, with or without radiation, to treat disease, to create room for donor stem cells, which replace a patient's lymphohematopoietic system, and to exert 
a graft versus leukemia effect on tumour cells to prevent relapse (Ezzone, 2013). In allogeneic HSCT, there is a separate donor and recipient for the hematopoietic progenitor cells. Autologous peripheral blood stem cell transplantation involves a rescue of patients' own hematopoietic stem cells from the effects of chemotherapy on their bone marrow. The patient has their stem cells collected and cryopreserved and then receives high-dose chemotherapy, which destroys their bone marrow. They subsequently receive their own stem cells back to restore hematopoietic function. This treatment allows the patient to be able to tolerate, haematologically, larger doses of chemotherapy than they could without the stem cell rescue (Ezzone, 2013). In autologous HSCT, the patient is both the donor and the recipient of the hematopoietic progenitor cells. A transplant coordinator is a highly specialized nursing role requiring an understanding of hematological diseases, related treatments, and the trajectory of care for this patient population. The allogeneic transplant coordinator arranges the activities related to the patient's transplant, taking into consideration the patient's plan of care and standards including baseline recipient and donor testing within 30 days of the infusion date. The coordinator must be aware of quality of care standards from FACT and provincial Cancer Care Ontario (CCO) benchmarking. Transplant coordination requires specialty trained nurses to enact this role. It is important that these nurses are appropriately orientated to their new role to effectively provide this unique patient care. Currently there is a gap in the literature exploring orientation practices for the HSCT coordination role.

The purpose of this study was to explore if a learning pathway would effectively support the competency-based orientation process for nurses new to the transplant coordination role at HHS. This orientation program is learner focused rather than instructor focused and guided by the learning pathway. This study focused on exploring the nurses' perception of a learning pathway, supported by a learning package and competency evaluation tool, during orientation to the transplant coordination role in the cellular therapy program at HHS. The research questions for the study were:

1. To what degree did the participants react favourably to the learning pathway?

2. To what degree did the participants acquire the knowledge regarding how to fulfill the role of transplant coordinator?

3. To what degree did the participants apply what they learned about transplant coordination in their new role?

Transplant coordination is an area that has not historically had a formalized learning pathway to guide orientation. At HHS, learning pathways were developed to provide a framework to assess learning needs of staff new to this area and to ensure that all necessary educational components are met. These pathways, informed by CANO (2002) practise standards and FACT standards, facilitate care for stem cell transplant patients by supporting staff new to the areas of both allogeneic and autologous stem cell transplant coordination. A self-directed learning package and competency evaluation tool for each sub-role of allogeneic and autologous stem cell coordination supports the pathways. The role of the learning packages is to help guide a nurse new to this area and serve as an adjunct to their learning assessment and plan. The role of the competency evaluation tool, completed by the learner in consultation with their preceptor and unit educator, is to provide a method to document and assess attainment of key competencies necessary in HSCT coordination.

\section{REVIEW OF LITERATURE}

Methods of orientation in the medical field were reviewed, as there is a lack of literature examining orientation to the nursing role of transplant coordination. Schumacher \& Risco (2017, p. 596) define competency as "an explicitly designed statement that encompasses a single or set of measurable or observable performances." Competency-based education has been the standard in the medical field for more than 20 years (Boyd et al., 2018). Greet et al. (2014) found that when new staff knew the criteria they were measured against, as is found in competency-based evaluation, they performed much better if these contained both practical and theoretical knowledge. The competency tool for the transplant coordination staff included the role and identified resources to which the new learner is expected to refer regarding the theoretical knowledge for that skill. Schumacher and Risco, (2017) note that shift from content- to competency-based orientation is outcomes based and relies on a "building-block" approach in which one skill is mastered then another is added; this approach allows a preceptor to be able to identify areas for which a new learner may require more support. As the role of transplant coordinator requires baseline disease-specific, content-based orientation, the learning package was developed to help guide the novice nurse with identifying learning needs and to provide resources in addition to specific training on transplant coordination processes and flow.

Milgore et al. (2017) note that comprehensive learning pathways improve employee satisfaction, increase morale, and promote staff retention. Johnston and Ferraro (2001) explain that a systemic and comprehensive clinical orientation is essential, and a pathway provides a framework for this orientation. A framework for orientation allows the new learner and preceptor to plan the focus of skill acquisition within specified timeframes utilizing criteria. If criteria are not met within specified timeframes, this can identify areas where a new learner is struggling (Johnston \& Ferraro, 2001). The unit's educator can work with the new learner and their preceptor to build a plan to close these gaps. Bumgarner and Biggerstaff (2000) view a learning pathway as a road map to guide a new learner and their preceptor; they note that learning pathways promote critical thinking, reduce reality shock in new roles, and increase both job satisfaction and retention. The effectiveness of incorporating a learning pathway to guide new learners is well established. Combining a learning pathway with competency-based learning promotes a design to prepare learners new to the transplant coordinator role and allow them to move beyond the novice role effectively. 


\section{DEVELOPMENT OF THE LEARNING PATHWAY}

Historically, the orientation program to the HSCT coordination role at HHS was solely preceptor-based without identified competencies, formal timelines, or an identified process to learn the various steps involved within this role. To build structure into the orientation to this role the learning pathway, supported by a learning package and a competency evaluation tool, was developed with input from experienced transplant coordination nurses. The complex process of transplant coordination was divided into multiple steps. As the novice nurse completes a step, and as they feel comfortable performing the tasks within this step, they move onto the next step. Each step incorporates time for the learner to observe their preceptor within the role and then for the learner to perform the roles with varying degrees of assistance. The learner gradually works more independently until they can complete a particular process with minimal to no assistance. The time to progress through various steps in the pathway varies based on learner needs as assessed in collaboration with the preceptor, although recommended timeframes exist to guide the learner's progression. The new learner maintains a competency evaluation tool, in consultation with their preceptor, tracking completion for each step in the pathway. Suggested resources and measurable outcomes support each step in the tool. The allogeneic (Figure 1) and the autologous coordination role (Figure 2) each have separate learning pathways.

Figure 1. Allogeneic Coordination Learning Pathway

\section{Orientation Pathway to Novice Allogeneic Transplant Coordinator}

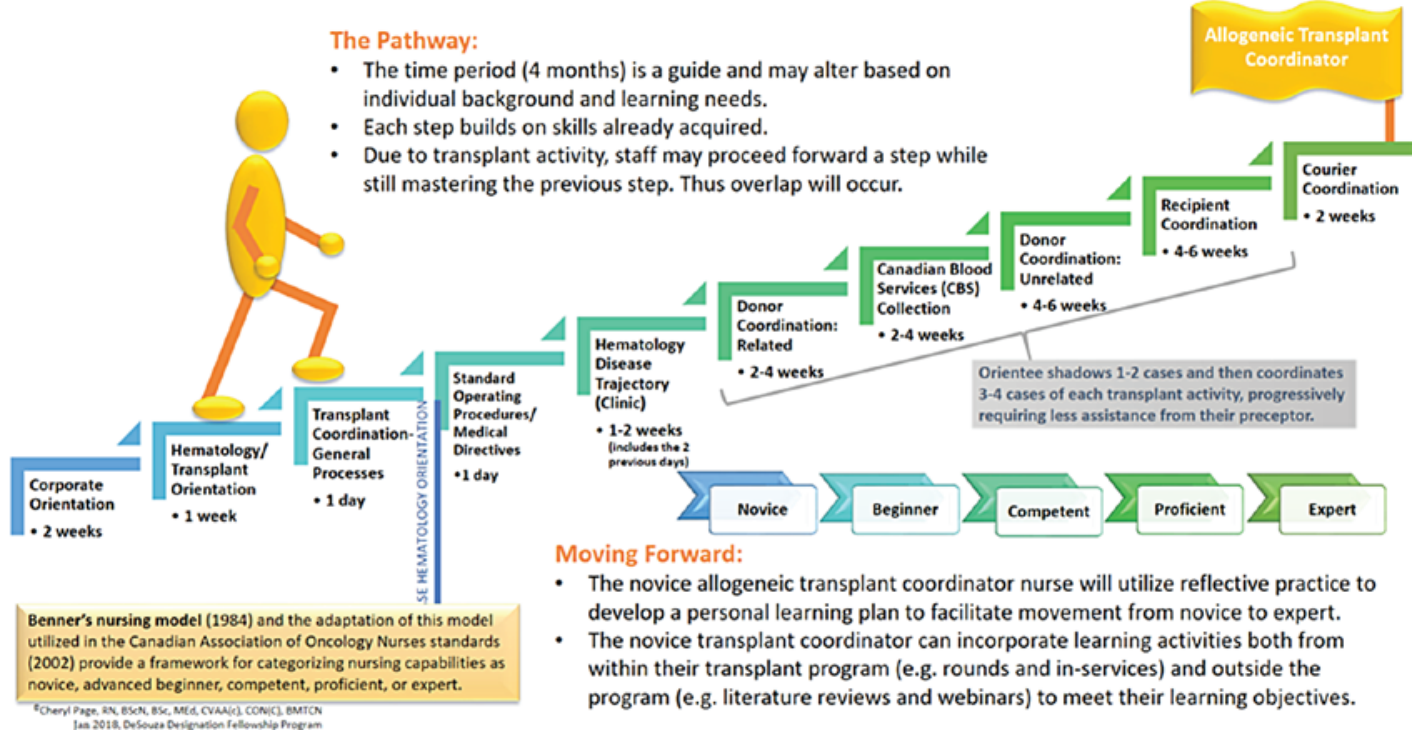

Figure 2. Autologous Coordination Learning Pathway

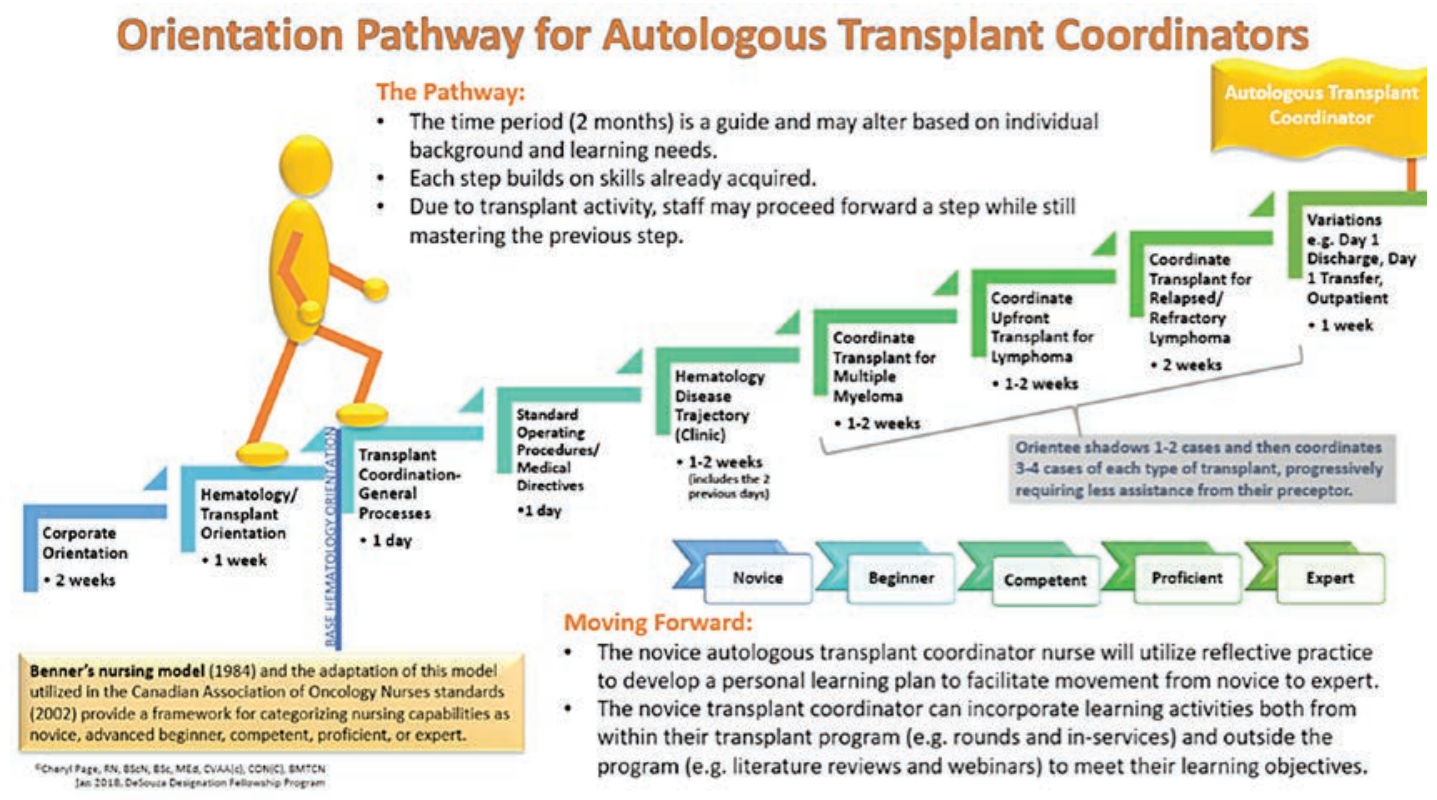


Benner's nursing model (1984) and the Canadian Association of Oncology Nurses standards (2002) provide a framework for categorizing nursing capabilities from novice, to advanced beginner, to competent, to proficient, and to expert. Benner's model (1984) acknowledges the roles both knowledge acquisition and practical skill gained through experience play in the education of a nurse. When an expert hematology nurse transitions to a new role such as a transplant coordinator, they revert to a lower competency level on Benner's model (1984) for that new role. A nurse may move through these stages faster in this new role if the nurse has transferable skills related to this area. The new transplant coordinator learning pathway is designed to prepare the nurse to move through these stages of competency.

The levels of Benner's model (1984) include novice, advanced beginner, competent, proficient and, ultimately, expert. In adapting Benner's model (1984) to the transplant coordination role, a novice nurse has conceptual understanding, minimal clinical experience, and limited knowledge of transplant coordination; the novice nurse seeks assistance when making clinical decisions. An advanced beginner nurse has conceptual understanding, minimal clinical experience, and some knowledge of transplant coordination; the advanced beginner nurse has limited exposure to clinical situations, but can identify normal findings (Benner, 1984). A competent nurse has conceptual understanding and is clinically experienced with skill performance; the competent nurse has had varied exposure to many situations, can identify normal and abnormal findings, can prioritize, and can manage complex situations (Benner, 1984). A proficient nurse has conceptual understanding, proficient performance, is clinically experienced, and can make quick and accurate clinical judgments; the proficient nurse has extensive exposure in most situations, can prioritize and can anticipate potential assessment changes (Benner, 1984). An expert nurse can synthesize and apply information, is highly skilled clinically, and has an extensive and well-developed knowledge; an expert nurse has extensive exposure with deep understanding of the situation, can rapidly and consistently identify actual and potential assessment changes, and can rapidly prioritize situations under all conditions (Benner, 1984).

\section{METHOD}

This study utilized a quasi-experimental non-equivalent time-series research approach using a convenience sample of participants.

\section{Participants}

The sample included nurses orientating to the transplant coordination role at HHS. During the period of this study, eight nurses completed orientation to either the allogeneic or the autologous transplant coordination role, all of which consented to take part in the study. If a nurse had orientated to a transplant coordination area in the past, allogeneic or autologous, and are now being orientated to the other stream, they were included in the study. This constitutes enrolment of $100 \%$ of this target population. Recruitment for this study included an information session explaining the intervention, and for those interested in the study, an informed consent obtained by a staff member skilled in obtaining informed consent and not involved in the operations of the coordination team.

The participants had a vast difference in nursing experience ranging from four to 30 years of nursing experience in hematology, with an average of 14 years. Hematology background experience included various work settings including inpatient care, outpatient care, apheresis unit, and outpatient clinic. Five of the participants orientated to the role of autologous transplant coordination and three to allogeneic transplant coordination. Two of the participants in this study had been orientated previously to the autologous coordination role and now were orientating to the allogeneic coordination role. None of the participants had previously orientated to the allogeneic role.

\section{Intervention}

Nurses were orientated using the learning pathway, competency evaluation tool, and learning package. The nurses were aware these tools would be utilized in the orientation process whether they decide to participate in this study or not. Staff participating in the study completed a preorientation and postorientation evaluation. The learning pathway was introduced as a guide for the orientation process. The staff received the learning package to facilitate knowledge transfer specific to the new role. A competency evaluation tool recorded and tracked the learner's progress.

\section{Measurements}

The Kirkpatrick model (2006) used in this study as a framework to evaluate training effectiveness, looks at four levels of evaluation. Level one of this model evaluates the degree to which participants reacted favourably to the training (Kirkpatrick, 2006). Level two evaluates the degree to which the participants acquired the intended knowledge, skills, and/or attitudes based on their participation in a training (Kirkpatrick, 2006). Level three evaluates the degree to which the participants applied what they learned during the training to their job (Kirkpatrick, 2006). Level four evaluates the degree to which the targeted objectives or outcomes occurred because of the training (Kirkpatrick, 2006). This final level was not formally evaluated in this study.

The evaluation on the effectiveness of the training program utilized surveys adapted from the Kirkpatrick model (2006). The surveys assessed Kirkpatrick (2006) evaluation levels one, two and three. It was beyond the scope of this study to measure level four, results-based outcomes, of the transplant coordination role.

\section{1) Level One: To what degree did the participants react favourably to the learning pathway?}

A satisfaction survey rated the ease of use of the learning pathway, the value of the information in the learning package, and the comprehensiveness of the competency evaluation tool. A five-point Likert-type scale was used with ' 0 ' indicating strongly disagree with each statement and ' 5 ' indicating strongly agree. This survey was administered immediately after the completion of the orientation program. 


\section{2) Level Two: To what degree did the participants acquire the knowledge of how to fulfill the role of transplant coordinator?}

Extent of knowledge about transplant coordination was assessed as a baseline preintervention and postintervention. A survey on self-reported knowledge covered four areas of the transplant process: how a HSCT is completed, how a HSCT fits into a patient's trajectory of care; the patient flow during a HSCT, and the coordination flow during a HSCT. Additionally, learners were asked in the survey to rate the value of the learning pathway, the learning package, and the competency evaluation tool in guiding their orientation. A five-point Likert-type scale was used with ' 0 ' indicating very low value with each statement and ' 5 ' indicating very high value. The learners completed the survey prior to the start of their orientation and immediately following completion of the orientation period.

\section{3) Level Three: To what degree did the participants apply what they learned about transplant coordination?}

A change in behaviour related to transplant coordination was assessed preintervention and postintervention. A survey on self-reported behaviour covered three areas of transplant coordination care: ability to provide patient education on the HSCT process, ability to coordinate the HSCT, and competency level based on Benner's (1984) model. A five-point Likerttype scale was used with ' 0 ' indicating very low ability with each statement and ' 5 ' indicating very high ability.

\section{STATISTICAL ANALYSIS}

A five-point Likert-type scale captured the participant's responses. There is debate regarding the use of a parametric analysis of Likert-like data versus a non-parametric analysis method (Sullivan \& Artino, 2013). Halter (2017) and Harpe (2015) state that the data resulting from a Likert-like scale of five points or higher is a measured, scaled difference between the response choices, thus the data can be considered continuous. Sullivan and Artino (2013) recommend comparison of means, as used in a $t$-test, rather than modes, as utilized in a signed rank test, for Likert-type data, as these parametric tests are more robust for Likert-type data than non-parametric if the sample size is at least 5 to 10 observations. Meek et al. (2007) extensively compared the parametric t-test to the non-parametric Wilcoxon signed rank test utilizing Likert-like data and found the $t$-test to have a higher power, even for very small sample sizes.

A parametric two-tailed paired t-test analysis assessed the difference between the mean scores, before and after the educational intervention. As an exploratory pilot study, a twotailed test can help determine the effectiveness of using a learning pathway to guide orientation program of a nurse new to transplant coordination. The content areas in each of the Kirkpatrick levels of evaluation were tested separately. Level one, reaction to the training program, was only measured post the intervention. For level two (knowledge) and level three (behaviour), the participant was asked the same questions pre- and postcompletion of the learning pathway. The means of these scores were compared to evaluate if any change had occurred.
The field of transplant coordination nursing is a sub-specialty of nursing in the already highly specialized area of hematology nursing. Majhail et al. (2015) reviewed the models of care at 85 stem cell transplant centres in the United States and found an average of four transplant coordinators at each site. Hertzog (2008) recommends that for pilot studies, at least 10\% of the population is needed. Due to the small number of coordinators in any given HSCT centre, the required sample size needed to be higher than $10 \%$ to gauge statistical significance of the results. Israel (2003) states that one approach to mitigate sampling errors and increase precision with small populations is to take a census, utilizing the entire population as the sample. In our study, we obtained $100 \%$ of the new staff orientating to the coordinator role. Therefore, the results of this pilot study demonstrate significant impact of our intervention at HHS and results can be used to inform further studies at other centres.

\section{RESULTS}

Eight staff consented to participate in the study and all eight staff completed the study. There was not a significant correlation with preassessment $(r=0.61, p=.112)$ or postassessment $(r=0.42, p=.304)$ of transplant coordination nursing competency and years of experience in hematology. There was also not a significant correlation with preassessment $(r=0.19$, $p=.677)$ or postassessment $(r=0.30, p=.513)$ of transplant coordination nursing competency and experience in a previous different coordination role.

\section{First Level of Evaluation: Reaction}

The first level of evaluation measured the degree to which the participants of the study reacted favourably to the learning pathway and the related education supports (intervention). The postintervention survey was completed by all eight of the participants. The results indicate that $100 \%$ of participants reacted favourably to the learning pathway such that $37.5 \%$ $(3 / 8)$ of the participants strongly agreed and a further $62.5 \%$ $(5 / 8)$ agreed that the learning pathway was easy to follow. The survey also found that $75 \%(6 / 8)$ of the participants strongly agreed and a further $25 \%(2 / 8)$ agreed that the learning package included valuable information and resources. Additionally, $62.5 \%(5 / 8)$ of participants strongly agreed and a further $37.5 \%$ $(3 / 8)$ agreed that the competency-based evaluation encompassed all aspects of the role.

\section{Second Level of Evaluation: Knowledge}

A paired-samples $t$-test was conducted to evaluate the impact of the educational intervention on the staff's scores for four areas of knowledge. For self-reported rating on knowledge of how transplants are performed, there was a statistically significant increase in these scores from the preevaluation $(\mathrm{M}=3.5, \mathrm{SD}=0.53)$ to the postevaluation $(\mathrm{M}=4.5, \mathrm{SD}=0.53)$, $\mathrm{t}(7)=3.74, p=.007$. The eta-squared statistic $(0.7)$ showed a large effect.

For self-reported rating on knowledge of the transplant role in the patient's trajectory of care, the improvement was also significant from the preevaluation $(\mathrm{M}=3.25, \mathrm{SD}=0.46)$ to the postevaluation $(\mathrm{M}=4.63, \mathrm{SD}=0.52), \mathrm{t}(7)=7.51, p<.001$, eta squared equals 0.9 . 
For self-reported rating on knowledge of the patient flow during the transplant process, there was a statistically significant increase in these scores from the preevaluation $(\mathrm{M}=2.88$, $\mathrm{SD}=0.64)$ to the postevaluation $(\mathrm{M}=4.50, \mathrm{SD}=0.53)$, $\mathrm{t}(7)=6.18, p<.001$, eta squared equals 0.8 .

For self-reported rating on knowledge of the transplant coordination flow during the transplant process, the increase in these scores was significant from the preevaluation $(\mathrm{M}=1.75, \mathrm{SD}=0.71)$ to the postevaluation $(\mathrm{M}=4.38$, $\mathrm{SD}=0.74), \mathrm{t}(7)=9.98, p<.001$, eta squared equals 0.9 .

Within the knowledge assessment, the learners were asked to rate pre- and postintervention how they felt the learning pathway and the supporting resources, a learning package and competency assessment form, helped to guide their orientation. For self-reported rating on value of the learning pathway, there was no change from the preevaluation $(M=4.63$, $\mathrm{SD}=0.52)$ to the postevaluation $(\mathrm{M}=4.63, \mathrm{SD}=0.52), \mathrm{t}(7)=0$, $p=$ n.s. The self-reported rating on value of the learning package also showed no change from the preevaluation $(\mathrm{M}=4.75$, $\mathrm{SD}=0.46)$ to the postevaluation $(\mathrm{M}=4.88, \mathrm{SD}=0.35)$, post evaluation, $\mathrm{t}(7)=0.55, p=$ n.s. For self-reported rating on value of the competency evaluation tool, there was not a statistically significant increase in these scores from the preevaluation $(\mathrm{M}=4.75, \mathrm{SD}=0.46)$ to the postevaluation $(\mathrm{M}=4.50$, $\mathrm{SD}=0.53$, post evaluation), $\mathrm{t}(7)=0.80, p=\mathrm{n} . \mathrm{s}$.

On the preassessment, the participants rated the potential value of the pathway as high, with $37.5 \%$ agreeing and $62.5 \%$ strongly agreeing that the pathway would help guide their orientation. The postassessment had the same results with the participants rating the actual value of the pathway as high. Likewise, for the supporting documents, 25\% agreed and 75\% strongly agreed that the learning package would guide their orientation. In the postevaluation, this rose further as $12.5 \%$ agreed and $87.5 \%$ strongly agreed that the learning package would guide their orientation. This increase, however, was not a statistically significant one. For the competency assessment tool, $25 \%$ agreed and $75 \%$ strongly agreed that this tool would help guide their orientation prior to orientation. The postassessment saw a decrease to $50 \%$ agreed and $50 \%$ strongly agreed, but this was not a statistically significant change. The participants anticipated that the learning pathway and supporting tools would help guide their orientation and this view did not change following orientation.

\section{Third Level of Evaluation: Behaviour}

A paired-samples $t$-test was conducted to evaluate the impact of the educational intervention on the staff's scores for three behaviours. For self-reported rating on ability to provide patient education on the transplant process, there was a statistically significant increase in these scores from preevaluation $(\mathrm{M}=2.63, \mathrm{SD}=1.06)$ to postevaluation $(\mathrm{M}=4.88, \mathrm{SD}=0.35)$, $\mathrm{t}(7)=6.15, p<.001$. The eta-squared statistic $(0.8)$ showed a large effect.

For self-reported rating on ability to coordinate each step of the transplant process, there was a statistically significant increase in these scores from preevaluation $(\mathrm{M}=1.75$, $\mathrm{SD}=0.71)$ to postevaluation $(\mathrm{M}=4.75, \mathrm{SD}=0.46), \mathrm{t}(7)=11.22$, $p<.001$. The eta-squared statistic (0.9) showed a large effect.

For self-reported rating of nursing competency based on Benner's Model, there was a statistically significant increase in these scores from preevaluation $(\mathrm{M}=1.75, \mathrm{SD}=0.71)$ to postevaluation $(\mathrm{M}=3.50, \mathrm{SD}=0.93), \mathrm{t}(7)=7, p<.001$. The etasquared statistic (0.9) showed a large effect.

\section{DISCUSSION}

In this pilot study on a HSCT coordinator orientation program, the participants reacted favourably to the learning pathway and to the supporting learning package and competency evaluation tool (Figure 3). In assessing the role of demographics on the results, it was found that neither years of previous experience in hematology nor experience in a different coordination role had a significant effect on competency levels prior to or following the intervention. This is likely due to the unique nature of these roles. All the participants had some

Figure 3. Kirkpatrick Level 1 Evaluation: Reaction

\section{Kirkpatrick Level 1 Evaluation: Reaction}

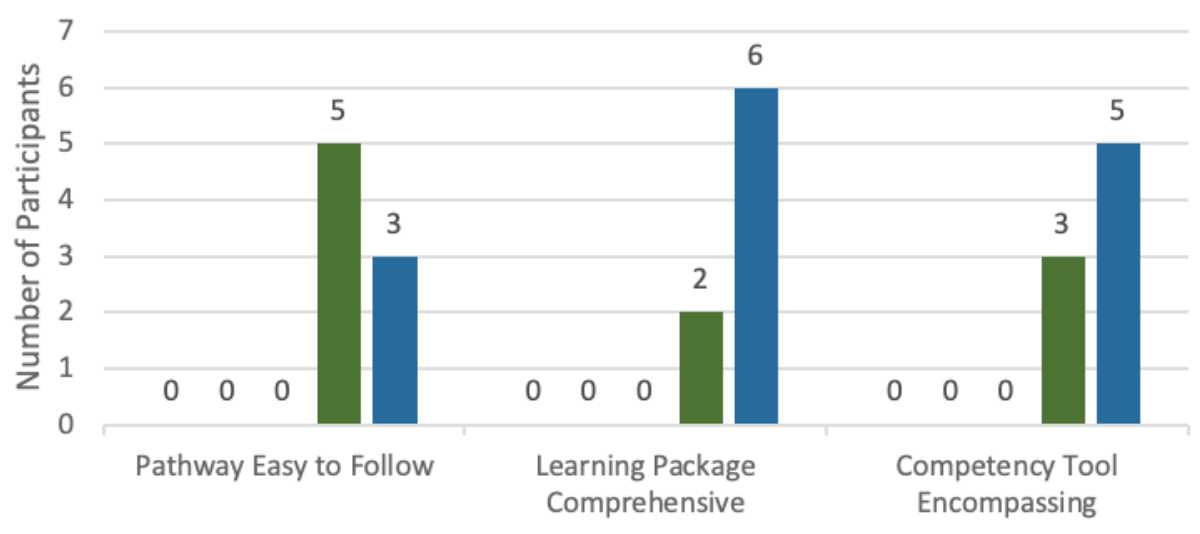

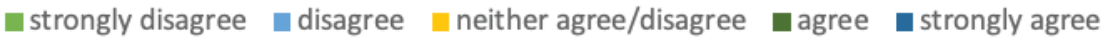


level of hematology nursing experience. Therefore, there was very little variance. If participants had no experience at all in this area, the results might be different.

The results of this study showed a significant shift in knowledge in key areas required for HSCT transplant coordination including: how a HSCT is completed, how a HSCT fits into a patient's trajectory of care, the patient flow during a HSCT, and the coordination of care for the donor and recipient during a HSCT (Figure 4). Examining the effect of the orientation program resources on the knowledge acquisition, the learners rated the expected value before orientation and the actual value after orientation of the learning pathway as high (Figure 5). The learners rated the potential value of the learning package high before orientation and even higher after orientation (Figure 5). The learners rated the potential value of the competency evaluation tool as high both pre- and postorientation (Figure 5). There was not a statistically significant difference of the value placed on these resources pre- and postorientation. One plausible explanation is that the expectation of the learning pathway, learning package and competency tool was already high at baseline, leaving very little room to improve (a phenomenon known as "ceiling effect"). This topic could warrant additional investigation.

There was a significant shift in behaviour pre- and postorientation, as demonstrated by an increase in three key areas of transplant coordination care: ability to provide patient education on the HSCT process, ability to coordinate the HSCT, and in nursing competency level based on Benner's

Figure 4. Shift in knowledge pre- and postorientation program.

\section{Kirkpatrick Level 2: Knowledge Pre and Postintervention}

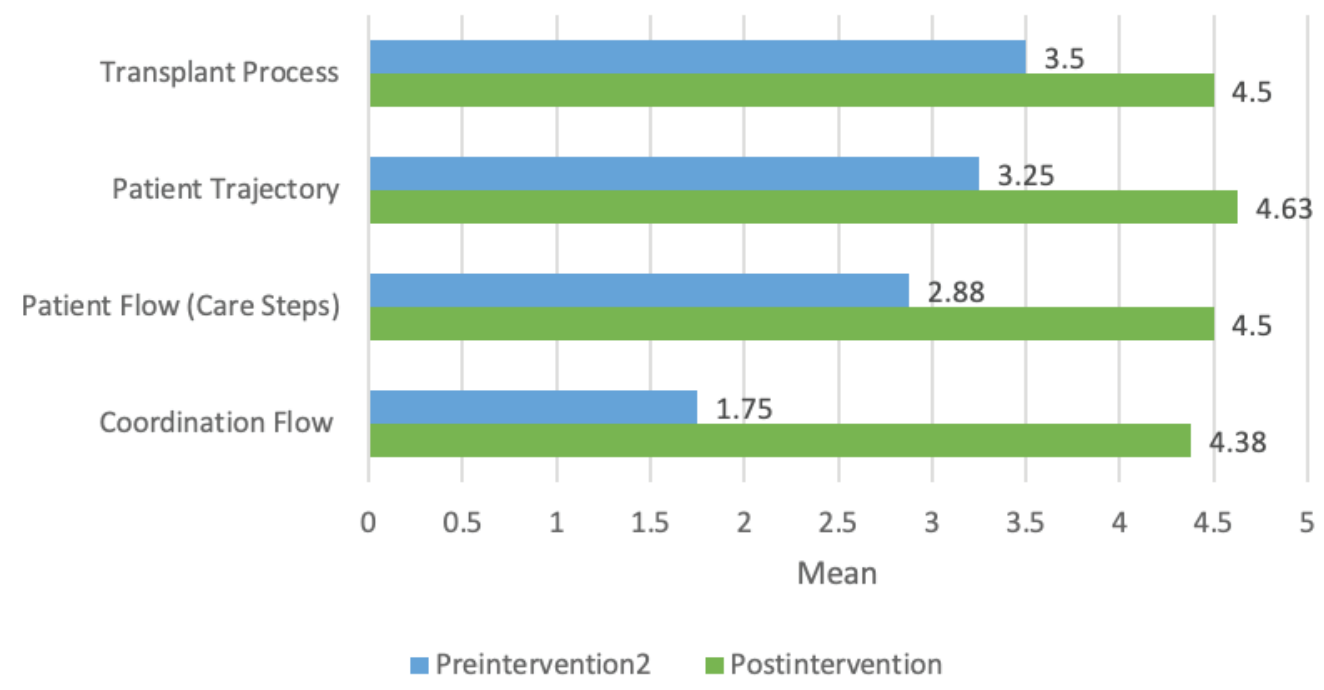

Figure 5. Rating of the value of the learning pathway, learning package, and competency evaluation tool pre- and postorientation

Value Placed on the Learning Pathway and Tools

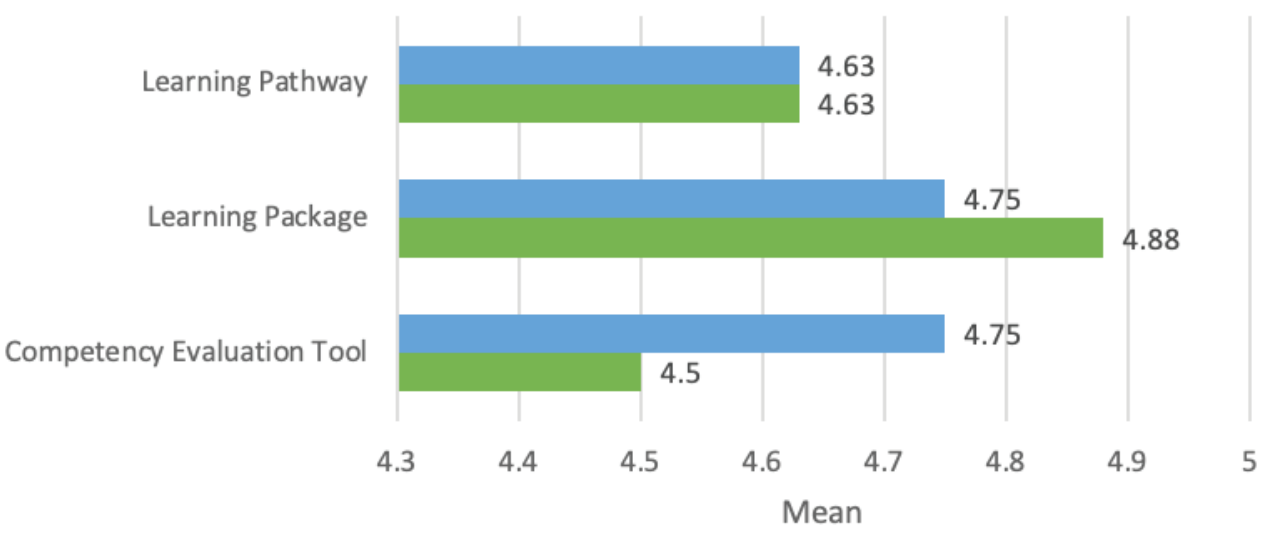

a Pre-Intervention2 a Post-Intervention 
model (1984) (Figure 6). The learners mostly scored themselves as novice or advanced beginner preorientation (Figure 6). Post orientation, most of the learners scored themselves as competent (Figure 7). The new HSCT coordinators will continue to advance through the stages of Benner's (1994) model as they gain further experience in their coordination role.

\section{Limitations}

There are a few limitations in this study. The fourth level of evaluation in the Kirkpatrick model (2006), which looks at the degree to which outcomes were affected based on the intervention, was not included in this study. Examining the impact of the orientation program on measurable outcomes within the HSCT would make a more robust study. However, as there are multiple factors that play a role in outcomes for this complex area, it would be difficult to isolate an effect directly related to orientation of the coordination staff. Further methods to strengthen the study could include additional evaluation of the participants four to six months following orientation to measure sustainability of knowledge and skills. The addition of a qualitative component to the evaluation could elicit an in-depth understanding of the participant's perceptions about the benefits of the orientation program over time.

Other factors could influence the learner's increase in knowledge and behaviour pre- and postorientation. Examples include preceptor factors or other continuing educational activities that a learner initiates on her own during this orientation period. Further studies evaluating the use of a learning pathway in HSCT orientation would assist in clarifying this.

This study utilized self-reporting for gathering data. Selfreporting can lead to bias, as respondents tend to inflate responses (Leggett et al., 2016). To help mitigate this effect, the study utilized the same survey questions pre- and postintervention and analysed the magnitude of change of over time. In addition, the survey was anonymous to reduce potential effects of peer pressure.

Figure 6. Shift in behaviour pre- and postorientation program

\section{Kirkpatrick Level 3: Behaviour Pre- and Postintervention}

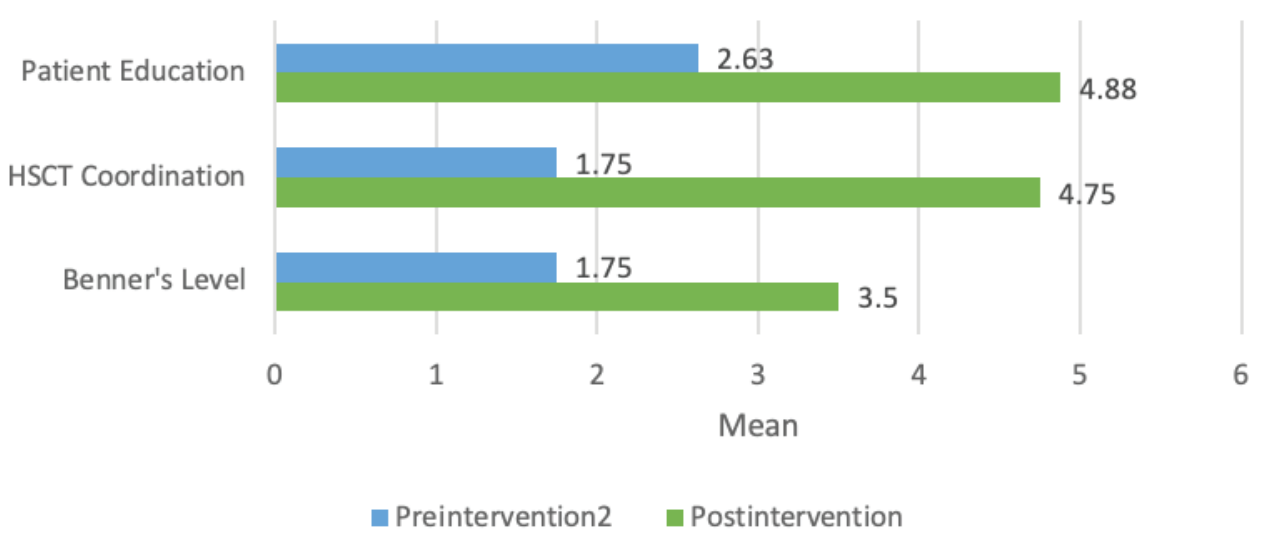

Figure 7. Change in Benner's Model competency stage pre- and postorientation program.

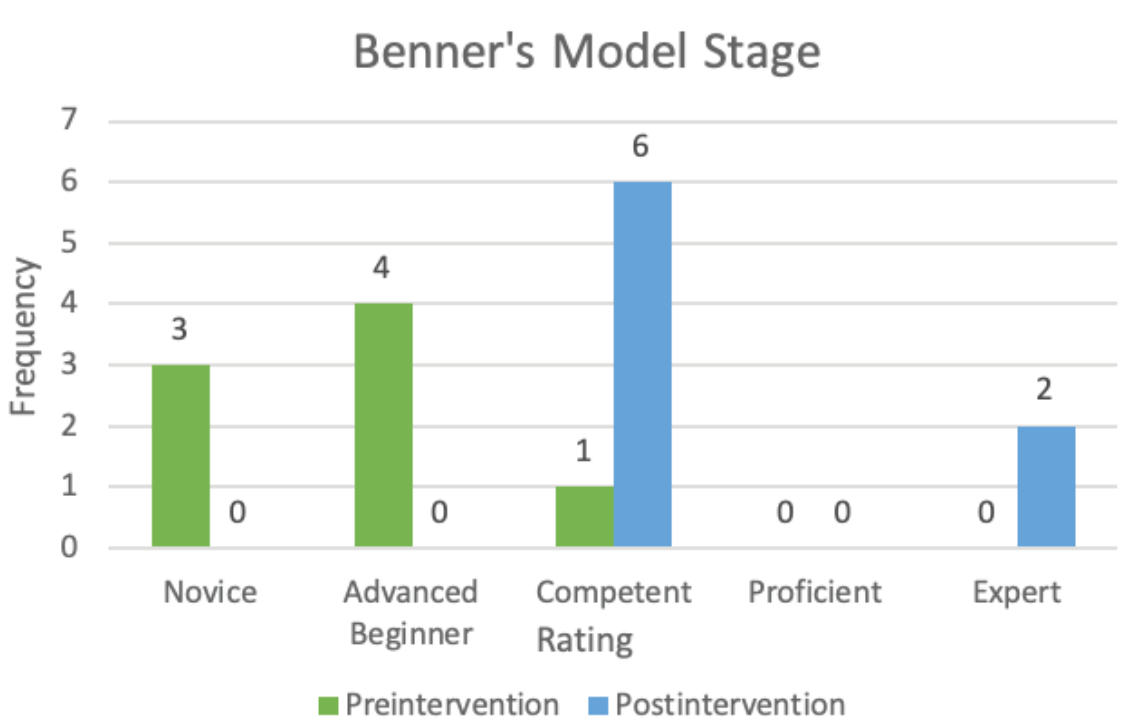


The self-reported ratings on knowledge include the following areas: 1) how transplants are performed; 2) the tranplant role in the patient's trajectory of care; 3) patient flow during the transplant process; and 4) transplant coordination flow. Each area was based on a Likert-like scale response to a single statement. The use of multiple statements to describe each knowledge area may improve the sensitivity of the measure to capture change. For example, instead of one statement about knowledge around how transplant is performed, this topic could be expanded by inquiring about more specific aspects of HSCT such as preparation, infusion, immediate complications, and late complications.

Finally, this study includes eight nurses at one HSCT centre, a relatively small sample size. However, this is typical in a highly specialized practice area such as HSCT. The study findings will need to be replicated by other HSCT centres.

\section{CONCLUSION}

The purpose of this study was to evaluate the effectiveness of a learning pathway when used to orientate nurses new to the transplant coordination role at HHS. The results indicate that the utilization of a learning pathway is an effective method to increase knowledge and behaviour of nurses orientating to the coordination role. The learners perceived their level of

\section{REFERENCES}

Benner, P. (1984). From novice to expert: Excellence and power in clinical nursing practice. Addison-Wesley.

Boyd, V. A., Whitehead, C. R., Thille, P., Ginsburg, S., Brydges, R., \& Kuper, A. (2018). Competency-based medical education: The discourse of infallibility. Medical Education, (1), 45. https://doi. org/10.1111/medu.13467

Bumgarner, S., \& Biggerstaff, G. (2000). A patient-centered approach to nurse orientation. Journal for Nurses in Staff Development, 16(6), 249-256.

Canadian Association of Nurses in Oncology. (2002). Practice standards and competencies. http://c.ymcdn.com/sites/cano. malachite-mgmt.com/resource/resmgr/standards/CONEP_ Standards2006September.pdf

CCO Complex Malignant Hematology Hematopoietic Cell Therapy Consultation Group (2017). Complex malignant hematology services in Ontario June 2017 - Year in review. https://www.cancercare.on.ca/ common/pages/UserFile.aspx?fileId=381199

Ezzone, S. (2013). Hematopoietic stem cell transplantation: A manual for nursing practice (2nd ed.). Oncology Nursing Society. ISBN: 9781935864196.

Fastre, G. M. J., van der, Klink, M. R., Amsing-Smit, P., \& van Merriënboer, J. J. G. (2014). Assessment criteria for competencybased education: A study in nursing education. Instructional Science, 42(6), 971-94.

Greet M. J., F., Marcel R. van der, K., Pauline, A., \& Jeroen, J. G. van, M. (2014). Assessment criteria for competency-based education: a study in nursing education. Instructional Science, (6), 971.

Halter, C.P. (2017). The PSPP Guide: An introduction to statistical analysis. Creative Minds Press Group.

Harpe, S.E. (2015). How to analyze Likert and other rating scale data. Currents in Pharmacy Teaching Q Learning, (7), 836-850.

Hertzog, M. A. (2008). Considerations in determining sample size for pilot studies. Research in Nursing \& Health, 31(2), 180-191. https:// doi.org/10.1002/nur.20247

Israel, D. G. (2003). Determining sample size. University of Florida IFAS Extension. https://www.tarleton.edu/academicassessment/ documents/Samplesize.pdf proficiency increased in this new role, according to Benner's (1984) new skill acquisition model in nursing, following the learning pathway-based orientation. The supporting orientation documents, the learning package and competency evaluation tool, positively affected this outcome.

Transplant coordination is an actively expanding role within many transplant programs, as the need for hematopoietic progenitor cell transplants increases. This specialty area has not previously had formal orientation programs. Drawing on the methods found to be successful in other fields of medical and nursing orientation, this study found use of a transplant coordination learning pathway, supported by a learning package and competency evaluation tool, significantly increased staff knowledge and proficiency level at one HSCT centre. Further studies are recommended to evaluate the effectiveness of the learning pathway-based orientation in this specialty area and assess the generalizability of these results to broader applications by replicating this study at other HSCT centres.

\section{ACKNOWLEDGEMENT}

We would like to acknowledge Tammy DeGelder, RN(EC), for her contributions during the participant consent process of this research project.

Johnston, P., \& Ferraro, C. (2001). Application of critical pathways in the maternity nursing orientation process. Journal or Nurses in Staff Development, 17(2), 61-66.

Kirkpatrick, D. L. (2006). Evaluating training programs: The four levels ( $3^{\text {rd }}$ edition). Berrett-Koehler.

Leggett, L. E., Khadaroo, R. G., Holroyd-Leduc, J., Lorenzetti, D. L., Hanson, H., Wagg, A., Padwal, R., \& Clement, F. (2016). Measuring resource utilization: A systematic review of validated self-reported questionnaires. Medicine, 95(10), e2759. https://doi.org/10.1097/ MD.0000000000002759

Majhail, N. S., Mau, L. W., Chitphakdithai, P., Payton, T., Eckrich, M., Joffe, S., Lee, S. J., LeMaistre, C. F., LeRademacher, J., Loberiza, F., Logan, B., Parsons, S. K., Repaczki-Jones, R., Robinett, P., Rizzo, J. D., Murphy, E., \& Denzen, E. M. (2015). Clinical research: Supportive care: National survey of hematopoietic cell transplantation center personnel, infrastructure, and models of care delivery. Biology of Blood and Marrow Transplantation, 2113081314. https://doi.org/10.1016/j.bbmt.2015.03.020

Miglore, G., Boeding, M., Barut, J., \& Harris, L. (2017). Nurses initiating change: Engaging nurses in development of unit orientation pathways and processes at an inpatient psychiatric hospital. Journal of The American Psychiatric Nurses Association [serial online], 23(4), 297-298. Science Citation Index.

Meek, G., Ozgur, C., \& Dunning, K. (2007). Comparison of the t vs. Wilcoxon signed-rank test for Likert scale data and small samples. Journal of Modern Applied Statistical Methods, 6(1), 91-106.

Schumacher, G., \& Risco, K. (2017). Competency-based nurse practitioner education: An overview for the preceptor. Journal for Nurse Practitioners, (9), 596. https://doi.org/10.1016/j. nurpra.2017.07.020

Sullivan, G. M., \& Artino, A. R., Jr. (2013). Analyzing and interpreting data from Likert-type scales. Journal of Graduate Medical Education, 5(4), 541-542. https://doi.org/10.4300/JGME-5-4-18 